\title{
Development of Molecular based Communication Protocols for Nanomachines
}

\author{
Frank Walsh ${ }^{2}$, Sasitharan Balasubramaniam ${ }^{1}$, Dmitri Botvich ${ }^{1}$, William Donnelly ${ }^{1}$, Sergey Sergeyev ${ }^{2}$ \\ Telecommunication Software and Systems Group ${ }^{1}$ \\ Waterford Institute of Technology ${ }^{1,2}$ \\ Carriganore Campus, Ireland \\ \{sasib, dbotvich,wdonnelly\}@tssg.org \\ $\left\{\right.$ fwwalsh, ssergeyev\}@wit.ie ${ }^{2}$
}

\begin{abstract}
Communication between nanomachines (e.g. biological nanoscale devices) has the potential to open up new opportunities and applications, especially in areas such as health care and information processing. Inspired by current data communication protocols, we propose a molecular-based communication protocol to enable reliable communication between nanomachines. In this paper, we present a protocol stack which enables nanomachine communication through the encoding and transmission of data as biomolecules. Extending previous work in logical nanocomputation, we define an error-recovery process through the use of a chemical state machine based on nano-logic circuit. Finally, we present initial results of simulation of nanomachine communication in the presence of both channel and chemical instability and demonstrate the performance of the nano-circuit in such conditions.
\end{abstract}

\section{Keywords}

Nanomachine, Nano-Communication, Communication Protocols, Molecular Communication, Nano-Computation

\section{INTRODUCTION}

The increased focus on nanotechnology in recent years has accelerated medical research towards the use of nano-devices in medical applications. These medical applications include the use of nano-scale devices to develop new drug delivery methods, cancer detection, diagnosis and treatment, assembly of macromolecules, and monitoring and supporting regulation within the organism. The focus of our research is on the development of communication protocols for nano-devices based on reusing protocols from telecommunications and data networks.

Nanodevices are small scale devices that are manipulated from materials of one billionth of a meter size [7]. Nanotechnology has progressed beyond plain particles with embedded chemicals to where new nanomachines are emerging with nano-computing

Nano-Net 2007 September 24-26, 2007, Catania, Italy. Copyright 2007 ICST ISBN 978-963-9799-10-3 DOI 10.4108/ICST.NANONET2007.2239 capabilities. A good example is nanorobots, where [1] developed the concept of nanorobots to assemble macromolecules. However, a key requirement in ensuring the efficient functionality of nanomachines is the cooperative capabilities between the various devices, due to their sheer size and processing power. To support this capability a key requirement is communication mechanisms between the various disparate nanodevices. Since nanodevices have limited processing capability, functionalities must be performed in cooperation with their environment. In this paper we propose a communication protocol for nanomachines in a biological medium. Our solution is based on a protocol stack which allows the nanomachines to encode the data bits into biomolecules, and support transport protocol such as transmission and error recovery through the control of nano-logic computation manipulated within biological cells. In this paper we focus particularly on investigating the use of transmission and error recovery protocol from data communications using molecular nano-logic computing. An example application of our proposed communication protocols is for communication amongst nanorobots [1]. This capability can be achieved by enabling nanorobots to migrate and attach themselves to cells and control the logic operations for communications. The robots can then migrate and interface to a different cell and perform the same communication operation.

The paper is organised as follows: Section 2 presents the related work on molecular computing and communication. Section 3 presents our overall solution, while section 4 presents some simulation results to illustrate our idea. Lastly section 5 presents the conclusion.

\section{RELATED WORK}

\subsection{Molecular Computing}

Stetter et al [4] developed a mechanism to allow logical nano computation to be performed by manipulating the concentration of enzyme molecules. Manipulation of enzymes includes mechanism of kinase activation to allow an enzyme to be active, while de-activation is performed through phosphorylation of the enzymes. This leads the enzymes to exhibit bistability behavior. From this behaviour, a number of solutions were proposed by the authors (e.g. fan architecture, linear cascade) which allowed for logical computation to be performed as AND, OR, and NOT gates. 


\subsection{Molecular Communications}

Enomoto et al [2] have proposed a molecular communication solution for nano-scale communication for nano-devices. The molecular communication allows devices to encode and decode information into molecules and to transport this information to peer nano-devices. The solution is inspired by biological systems that communicate using molecules, where molecular communication using molecular motors was observed within a biological cell. The authors seek to develop an engineered molecular communication system employing various processes (encoding, sending, propagating, receiving, and decoding) and components (sender and receiver). The solution proposed by the authors uses molecular motors (e.g. kinesin, dynein), which are used to transport materials in eukaryotic cells along filaments referred to as rail molecules (e.g. microtubles), for controlled nanomachine communication.

Nakano et al [3] proposed a mechanism to allow intercellular communication between distant nanodevices using induced calcium signalling. The solution proposed by the authors exploits the current calcium signalling between cells, by modifying the frequency and amplitude of the calcium concentration. Through this mechanism, calcium wave propagation occurs which allows controlled intercellular communication. By interfacing a nanodevice to a specific cell, calcium communication can be formed by controlling the calcium production in the event the device has access to the cytosol of the cell. In the event that cell has no access to the cytosol, then the nanodevice can emit substances that can bind to specific receptor of the cell that can induce calcium production. The challenges relating to describing accurately a communication channel for the transmission of messages in 'wet' techniques are highlighted by Alfano et al [5] who state the 'most urgent question' for molecular communications is the characterization of the 'wet' communication channel. The authors point to the challenge posed by non-linear nature of the channel and the necessity for experimentation and computer simulation to address this.

\section{PROPOSED SOLUTION}

Our proposed solution is based on applying current data communication protocols and mapping these to molecular and chemical computation in biological medium. Fig. 1 illustrates our mapping mechanism.

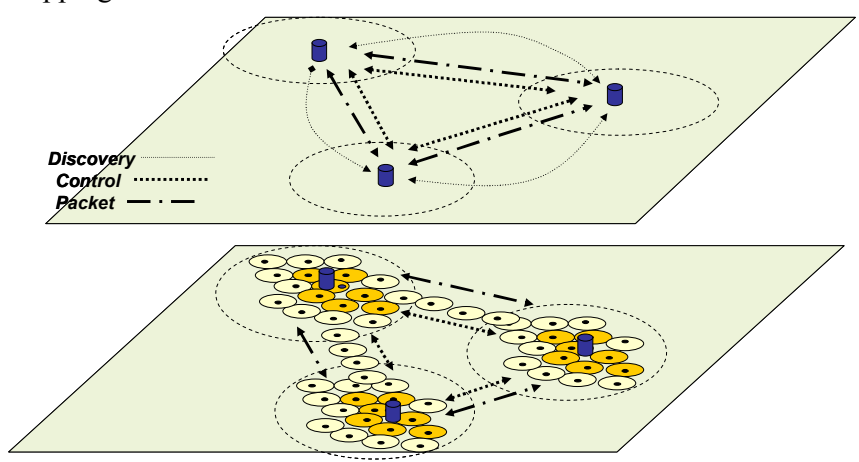

Figure 1. Overlay mapping of data networking and nano-machine communication in biological medium

As shown in Fig.1, plane A demonstrates how peer devices perform communication, where devices initially discover each other through messaging advertisement. Once devices are discovered (using ad hoc networking techniques), communication channels are established between the different devices using communication protocols to define how packet transmission can be established. Our intention is to map this process to biological medium in plane B, as shown in Fig. 1.

Our proposed solution is shown in Fig. 2, which is based on a bilayered protocol stack. Our application is based on the ability of nanomachines to bind to a cell and trigger logical functions to perform communication.

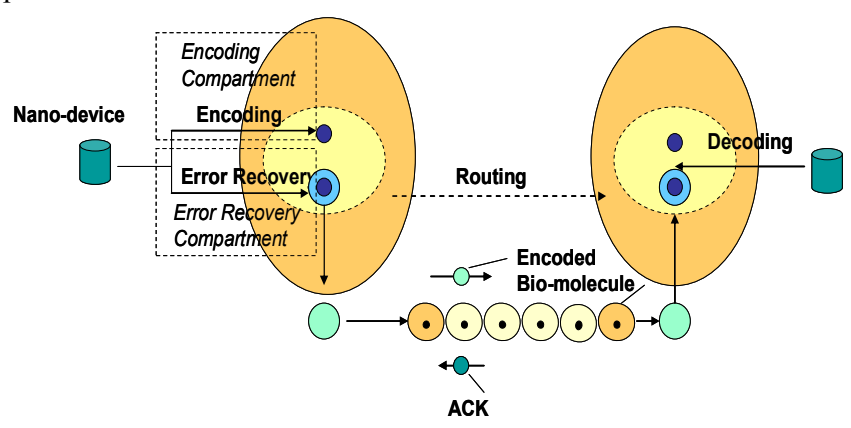

Figure 2. Communication protocol for nano-machines

The mappings to data communication are as follows: Application layer - Encoding and Transport layer- Transmission and ErrorRecovery. The proposed solution aims to perform each of these processes through interconnected compartments (Encoding compartments and Transmission and Error - Recovery compartments). Each of these compartments will hold nanological gates that perform the specific functionalities. Within these compartments are sub-compartments that hold specific nanogates, where the interconnection of these sub-compartments will produce a logic circuit. The subsections below will describe the processes of each compartment.

\subsection{Encoding}

A crucial requirement is the ability to encode the data into biomolecules and to manipulate the biomolecules when the data changes. Our mechanism of encoding information molecules is based on the concept of biomolecule computing, where certain biomolecular machines within the cell can act as automaton on encoded information [6]. A function of an automaton may include the ability to scan through a tape of symbols encoded in a biopolymer such as DNA strand, and modify certain symbols within the tape. An example of this process is illustrated in Fig. 3. The example of Fig. 3a shows a biomolecule that is segmented into an address string as well as a functionality string. By using state tables, shown in Fig. 3b, the manipulation of the string can be performed through rotation of state tables. Benenson and Shapiro [6] described the use of DNA and RNA polymerase, ribosomes, and recombinases that could be used to perform automaton functionalities. Through the defined state transition tables, the automaton process can scan specific cells of the tape and manipulate and change the encoded information (e.g. Fig. 3a shows how to change from address 1 to 2 , where cell 1 is changed by using the rules of the state transition and rotating through the address state table - Fig. 3b). This encoding process will be performed in the encoding compartment before transmitting to the Transmission and Error recovery compartment for transmission. 


\subsection{Transmission and Error Recovery}

Similar to the encoding process, the transmission error-recovery process is also based on using molecular computing techniques. Our solution is based on the solution proposed by Stetter et al [4]. The solution is based on defining a finite state machine that defines transmission and error-recovery at the transport layer (since we only have two layers, the transmission layer is also in charge of propagating the molecule at the physical layer where solutions of Enomoto et al [2] or Nakano et al [3] can be used.

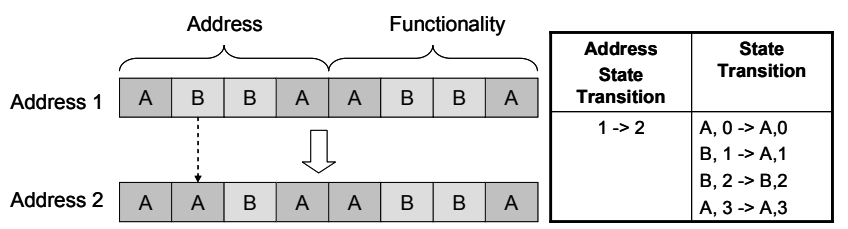

(A)

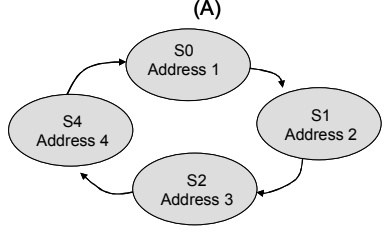

(B)

Figure 3. Nanomachine address and functionality encoding

The idea is based on defining the state machine and mapping this to a logic gate circuit. Once this is completed, a logic nano circuit is formed based on the control of various chemical concentrations based on Stetter's solution. For simplicity, we defined a simple transmission and error-recovery state machine illustrated in Fig. 4.

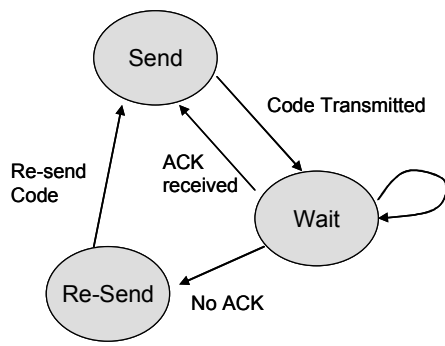

Figure 4. Error Recovery state diagram

Using the recurrent architecture for logical operations described by Stetter, the chemical state machine shown in fig. 4 can be modeled. In total, three different devices are modeled and simulated (And/Or/Not) and interfaced to create a logical circuit representing the state machine. The state machine modeled is a synchronous sequential system with behaviour that is dependant on the input and current state. Provision of a clock or cycle generation is provided by an independent chemical or enzymatic oscillation such as an autonomous calcium oscillation that resides in each molecular computer. The logic units are spatially subcompartmentalized with output species concentrations flowing to the input of the next gate through fixed channels. Conceptually, this could be thought of as a grid of gates interconnected by channels allowing the migration of output/input chemical concentrations.
Stetter discussed the implementation of logical units based on bio-molecules using a small recurrent architecture. The basis for these logic units is the abstraction of biochemical operations catalysed by Kinases and Phosphatases [4]. In this paper we combine this abstraction of logical units with the state machine model in Fig.4 to construct a molecular computer that controls transport of messages between nanodevices. The basis for these logic units, which is devised by Stetter, is described by the following system of equations [4]:

$$
\begin{aligned}
& \frac{d Z_{1}}{d t}=\alpha_{1,1} Z_{1} \widetilde{Z}_{1}+\alpha_{1,0} Z_{0} \widetilde{Z}_{1}-\beta_{1,2} Z_{2} Z_{1},(1) \\
& \frac{d Z_{2}}{d t}=\alpha_{2,3} C_{3} \widetilde{Z}_{2}-\beta_{2,1} Z_{2} Z_{1},(2)
\end{aligned}
$$

For this molecular state machine, the input concentration, for each logic unit comprises of two inputs, $Z_{01}$ and $Z_{02}$. Therefore equation (1) can be rewritten in the form [4],

$$
\frac{d Z_{1}}{d t}=\alpha_{1,1} Z_{1} \widetilde{Z}_{1}+\left(\alpha_{1,0_{1}} Z_{0_{1}}+\alpha_{1,0_{2}} Z_{0_{2}}\right) \widetilde{Z}_{1}-\beta_{1,2} Z_{2} Z_{1} \text {, }
$$

The state machine provides for connection orientated communication between two nanodevices. This communication process involves the transmission of a stack of encoded biomolecules from one nano-device to the other analogous to the 'first in first out' (FIFO) stack memory used in conventional communication systems. As we are not concerned with windowing at this stage, transmission takes place one message at a time with no requirement for packet sequence numbers. Transmission of following message biomolecules does not take place until an acknowledgement molecule is detected indicating successful delivery of the message biomolecule. Thus, we can initially avoid the complexity of congestion control, window size, and window scaling inherent in more sophisticated solutions while ensuring delivery. Admittedly this takes place at the expense of efficiency. Therefore, if an acknowledgement is not detected within a specific wait period, the last message is re-sent and a recommencement of the wait for acknowledgement is initiated.

The wait period is controlled chemically by the gradual increase in the concentration of a chemical initiated on transition to the Wait state. Once the concentration exceeds a threshold concentration the delay expiry is chemically signalled and transition to the resend state occurs. This mechanism could be constructed using the chemical bistability properties used by the logic units. Routing is reliant on the encoding of the relevant source and destination nano-machine address in the transmitting bio-molecule or packet and will be the subject of future work.

\section{SIMULATION}

To illustrate our proposed solution for transmission and error recovery, we have performed some simulation for biomolecule transmission. The purpose of the simulation is to illustrate mechanism of nano-machine control of molecular machines to transmit and re-transmit biomolecules in the event of message lost. Unlike conventional data networks, nano communication is a more challenging task due to the effect of errors caused by both 
chemical disruption in parallel with transmission errors. This will be illustrated through the simulation.

We assume that the information has been encoded into biomolecules from the Encoding compartment and transmitted to the Transmission and Error Recovery compartment for transmission. Our mechanism for transmission is based on the finite state machine model in Fig. 4, which is created comprising of the three states and four transitions. Conventional analysis using truth tables and Karnaugh maps results in the logic circuit shown in Fig 5. Input states require two bits, $S_{0}$ and $S_{1}$ and four transition events also require 2 bits, $\mathrm{I}_{0}$ and $\mathrm{I}_{1}$. Each logic gate, which is a sub-compartment of the Transmission and Error Recovery compartment, is simulated using the recurrent architecture shown in Fig.5 (b) as specified by Stetter. For present purposes we will assume communication is across a channel where signal delivery success rate can be adjusted to verify the performance of the chemical state machine and simulate a noisy or lossy channel.

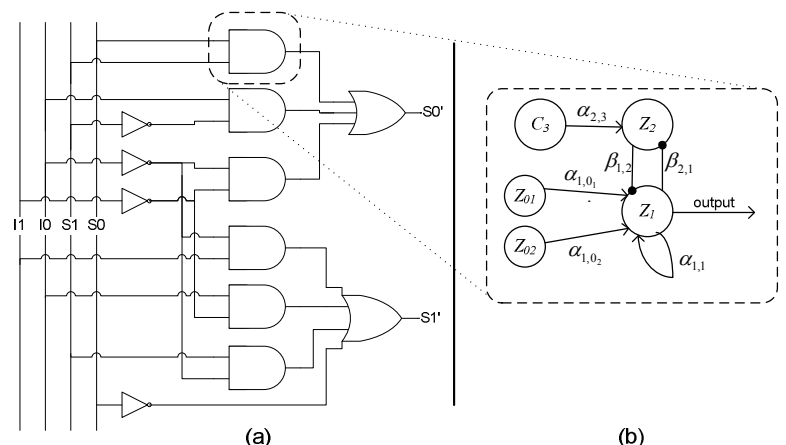

Figure 5. (a) Logic gate diagram of chemical state machine for transmission (b) Stetter's recurrent architecture that is the basis for logic gates.

In this simulation, initialization and input to the machine is performed by the associated nano-device after which it will attempt to successfully transmit ten messages to a destination device. If the machine enters the one possible unused state $\left(\mathrm{S}_{0}=0\right.$, $\mathrm{S} 1=0)$ then the nano-device will reinitialize the machine. Each simulation involves the transmission of ten packets to a destination device over a simulated channel with a loss rate based on a uniform distribution. It is assumed that packet transmission is the broadcast of encoded biomolecules and the distance between sending and receiving nanodevice is sufficient to permit intercellular communication. As described by Stetter we assume all activation rates are equal and that activation concentrations of input enzymes predominantly reside between 0 and 0.5 . The input into the AND and OR unit is a fan-in structure comprising of two input chemicals $\left(Z_{01}\right.$ and $\left.Z_{02}\right)$. Assuming the respective activation rates are constant for both $Z_{01}$ and $Z_{02}$, the input concentration $Z_{0}$ can be assumed to be the sum of both concentrations. In the case of an AND gate, two high input concentrations are required to push the system into the upper state of stability. Similarly, the OR gate enters the upper stability point on the occurrence of one high input concentration. Finally the NOT gate is identical to the OR gate except only one input species is considered and output is taken from $\mathrm{Z}_{2}$.

We also investigate the effect of chemical instability on performance of the chemical machine. The cause of this instability may be due to an unexpected flow of chemicals between subcompartments. This is simulated by altering the rate at which input chemical species $\left(Z_{0 x}\right)$ can activate $Z_{1}$. This is achieved by offsetting the specific activation rate at which input chemicals affect the active concentration of output $Z_{1}$. When this is confined to ability of input species to catalyse output species, equation (3) can be rewritten in the following form:

$$
\frac{d Z_{1}}{d t}=\alpha_{1,1} Z_{1} \widetilde{Z}_{1}+\left(\alpha_{1,0}+\varepsilon\right)\left(Z_{01}+Z_{02}\right) \widetilde{Z}_{1}-\beta_{1,2} Z_{2} Z_{1}
$$

where $\mathcal{E}$ is the magnitude of the disruption. This alters the location of the bistability region of the system and potentially cause the logic unit to function incorrectly. Also, modelling of both chemical noise and channel noise is a realistic assumption that these will be present in a real world system. Fig. 6 shows the output and input chemical concentrations of the logic subcompartment in Fig 5(b) for the transmission of ten packets over a lossless channel with $\varepsilon$ fluctuating randomly $\pm 0.2 \alpha_{1,0}$. As the input to this gate is $S_{0}$ and $S_{1}$, it enables the monitoring of the performance of the gate and the current state of the chemical state machine during the simulation. In ideal conditions, the state of the chemical machine continuously transitions between send state to wait state (logic ' 11 ' and logic ' 10 '). During the simulation, output of the gate begins to fluctuate and results in an incorrect output at about time $=90$. Following this, the machine incorrectly transitions to an unused state $\left(\mathrm{S}_{0}=0, \mathrm{~S}_{1}=0\right)$.
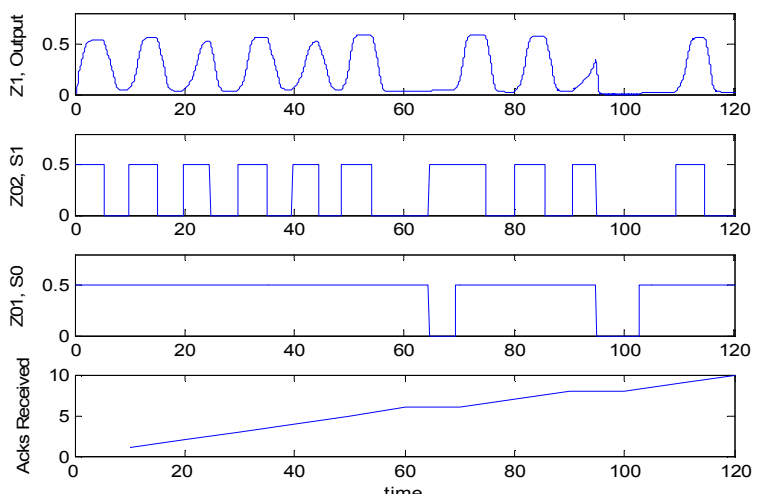

Figure 6. Simulation of AND gate highlighted in Fig. 5 during transmission of ten messages over a lossless channel. Top: trace of output signal $Z_{1}$. Second and third from top: Inputs $Z_{01}, Z_{02}$. Bottom: Acknowledged delivery of messages.

The bottom graph in Fig. 6 shows the effect of this instability on message transmission. The flat regions of this graph indicate message transfer error during simulation and correlates with incorrect machine states (through the simulation run, this resulted in two events). At time $=60$ the machine state incorrectly remains in a wait state instead of transitioning to resend or transmit state. At time $=90$, the machine incorrectly transitions to an unused state $\left(\mathrm{S}_{0}=0, \mathrm{~S}_{1}=0\right)$ after an incorrect output from the sub-compartment shown in fig. 5(b).

The average number of incorrect states due to changes in output activation for a sequence of ten simulations is shown in Fig. 7. Incorrect states begin to occur when the variation of the activation 
rate of $Z_{0}$ increases above $12 \%$ of its stable value. As expected, the number of incorrect state transitions increases significantly beyond this point. In order to investigate the environment for our nano-communication, the performance of the chemical machine was simulated in the presence of both chemical and channel uncertainty. Fig 8 shows the message transfer errors due to channel error rate of $10 \%$ and chemical error at $\varepsilon= \pm 0.2 \alpha_{1,0}$. The simulation of the chemical machine succeeds in delivering all twenty messages. This is accomplished due to the simplicity of the machine in that a message is only removed from the stack when an acknowledgement is successfully received. However, the simplified protocol also results in time in-efficiency.

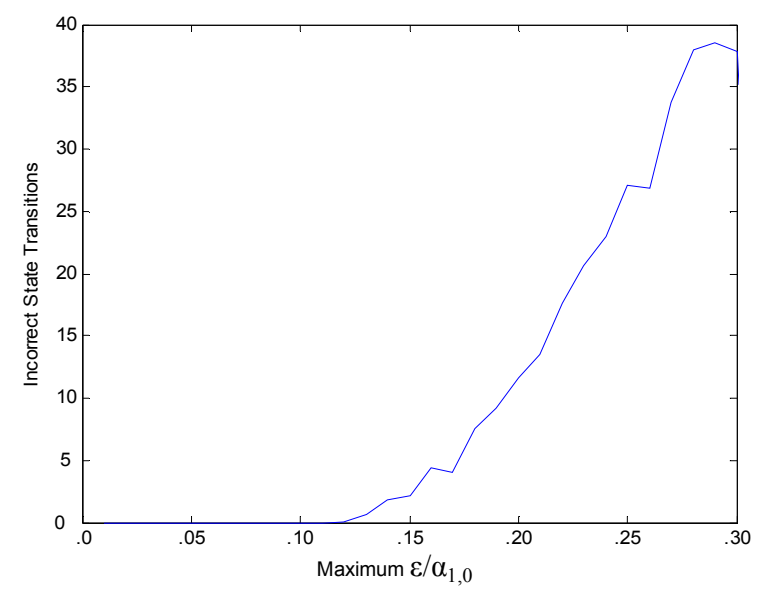

Figure 7. Occurrences of incorrect state transitions relative to the maximum value of $\varepsilon / \alpha_{1,0}$. Failures begin to occur at $\varepsilon / \alpha_{1,0} \approx 0.12$.

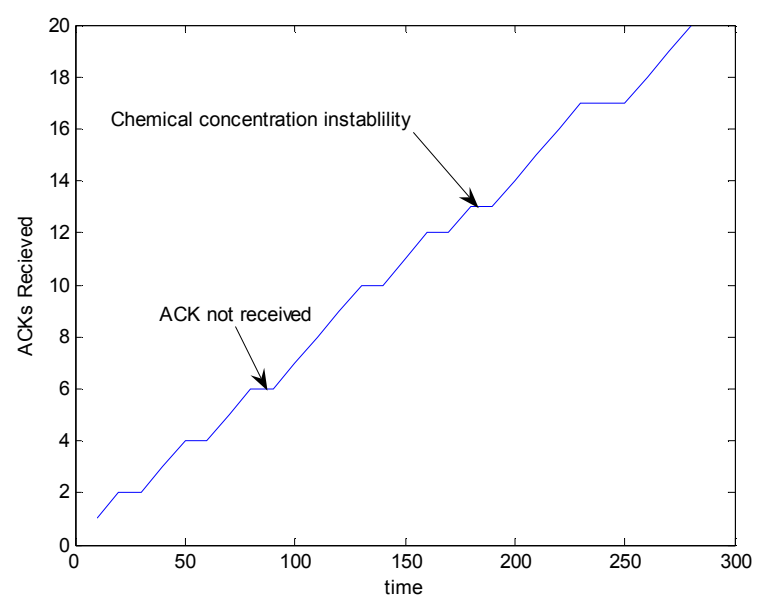

Figure 8. Transmission of 20 messages. Parameters: $\varepsilon= \pm 0.2 \alpha_{1,0}$, channel error rate $10 \%$

Our simulation has illustrated the challenge of communication for nanomachines. Unlike conventional computing devices, where packet loss can be due to lossy channels, in nanocommunication the biomolecule packet can be lost due to both the lossy channel as well as instability in logic operations at the end device. For our future work, we intend to investigate mechanisms to minimise logic operation errors due to chemical instability as well as investigate multiple packet transmission. We will investigate techniques to accomplish windowing and congestion control in a molecular setting. Furthermore, we will also investigate molecular communication interface between the transmitting device and the physical medium and evaluate the usage of current cell-cell signalling techniques such as calcium signalling.

\section{CONCLUSION}

We have investigated a protocol for reliable molecular based communication between nano-devices, which is based on the reuse of current telecommunications and data communication protocols. The proposed solution involves a bi-stack layer which comprises of compartmentalized nano-logic circuits. The two compartments corresponding to the bi-layers includes Encoding compartment as well as Transmission and Error Recovery compartment. The advantage of our solution is the ability for nanomachines to interface to a cell and control the logical operations for communication. This methodology is ideal for nanomachines which do not require communication capabilities and can control the cell to perform any communication. We have demonstrated the feasibility of this approach through the simulation of a chemical computation based nano-circuit to control transmission and error recovery. Initial simulation results have highlighted the inherent challenges for both chemical and channel instability present in the biological medium. We have shown the effects of both channel noise and unexpected chemical concentration variations on the performance of our transmission and error recovery process.

\section{References}

[1] A. Cavalcanti, R. A. Freitas Jr., "Nanorobotics Control Design: A Collective behaviour approach for medicine", IEEE Transaction on NanoBioscience, vol. 4, no. 2, June 2005

[2] A. Enomoto, M. Moore, T. Nakano, R. Egashira, T. Suda, A. Kayasuga, H. Kojima, H. Sakakibara, K. Oiwa, "A molecular communication system using a network of cytoskeletal filaments", 2006 NSTI Nanotechnology Conference, May 2006.

[3] T. Nakano, T. Suda, M. Moore, R. Egashira, A. Enomoto, K. Arima, "Molecular Communication for Nanomachines Using Intercellular Calcium Signalling”, IEEE Nano 2005, Japan, June 2005.

[4] M. Stetter, B. Schurmann, M. Hofstetter, "Logical NanoComputation in Enzyme Reaction Networks" in Proceedings of BIONETICS 2006, Cavalese, Italy 2006.

[5] G. Alfano, D. Morandi, "On Information Transmission Among Nanomachines”, Nano-Networks and Workshops, 2006

[6] Y. Benenson, E. Shapiro, "Molecular Computing Machines", Dekker Encyclopedia of Nanoscience and Nanotechnology, Marcel Dekker, 2004, pp. 2043 - 2055.

[7] G. A. Silva, "Introduction to Nanotechnology and its application to Medicine”, Surgical Neurology, 61, 2004, pp.216-220. 